The proof of Theorem 10.3 is similar, but with obvious modifications.

\title{
REFERENCES
}

1. Tibor Rad6, On convex functions, Trans. Amer. Math. Soc. vol. 37 (1935) pp. $266-285$.

2. H. B. Dwight, Tables of integrals and other mathematical data, Macmillan, 1934.

3. G. P6lya and G. Szegö, Aufgaben und Lehrsätze aus der Analysis, vol. 2, Berlin, Springer, 1925.

4. G. H. Hardy, J. E. Littlewood, and G. Pólya, Inequalities, Cambridge, 1934.

Emmanuel Missionary College

\section{A SIMPLE SUFFICIENT CONDITION THAT A METHOD OF SUMMABILITY BE STRONGER THAN CONVERGENCE}

\section{RALPH PALMER AGNEW}

1. Introduction. A matrix $a_{n k}$ of real or complex constants determines a transformation

$$
\sigma_{n}=\sum_{k=1}^{\infty} a_{n k} s_{k}
$$

and a method $A$ of summability by means of which a given sequence $s_{1}, s_{2}, \ldots$ is summable to $\sigma$ if the series in (1) converge and define numbers $\sigma_{1}, \sigma_{2}, \cdots$ such that $\sigma_{n} \rightarrow \sigma$ as $n \rightarrow \infty$. If a sequence $s_{n}$ is summable $A$, we say that $A\left\{s_{n}\right\}$ exists and that $s_{n}$ belongs to the summability field of $A$. If $s_{n}$ is summable $A$ to $\sigma$, we say that $A\left\{s_{n}\right\}=\sigma$. The method $A$ is regular if $A\left\{s_{n}\right\}=\lim s_{n}$ whenever $\lim s_{n}$ exists.

Toeplitz [1911] (reference in bibliography at end of this paper) proved that $A$ is regular if and only if the three conditions

$$
\begin{aligned}
\sum_{k=1}^{\infty}\left|a_{n k}\right| & \leqq M, & n=1,2,3, \cdots, \\
\lim _{n \rightarrow \infty} a_{n k} & =0, & k=1,2,3, \cdots, \\
\lim _{n \rightarrow \infty} \sum_{k=1}^{\infty} a_{n k} & =1 &
\end{aligned}
$$

Presented to the Society, November 24, 1945; received by the editors September 8 , 1945. 
are satisfied, $M$ being a constant depending on the matrix $a_{n k}$. Immediately thereafter, Steinhaus [1911] proved that no regular $A$ has a summability field containing all sequences; in fact, to each $A$ correspond sequences, whose elements are zeros and ones, which are nonsummable $A$. Actually, Toeplitz and Steinhaus considered only rowfinite transformations, but their methods give the facts stated.

An attempt to prove that each regular $A$ has a summability field containing some divergent sequences cannot succeed, because there exist regular methods $A$ whose summability fields are identical with the class of convergent sequences. Convergence is the simplest example. A classic theorem of Mercer [1907] and its generalizations, known as Mercerian Theorems, provide further examples. More examples of less special forms have been given by Agnew [1932], Sunouchi [1934] and Radó [1938]. It is the purpose of this paper to prove and discuss the following theorem and a generalization of it.

Theorem 1. If $A$ is regular and satisfies the condition

$$
\lim _{n, k \rightarrow \infty} a_{n k}=0
$$

then some divergent sequences of zeros and ones are summable $A$.

This theorem and the result of Steinhaus given above combine to yield the following theorem.

THEOREM 2. If $A$ is regular and satisfies (5), then some but not all divergent sequences of zeros and ones are summable $A$.

It is of course well known and obvious that if the matrix $a_{n k}$ has an inverse $\alpha_{n k}$, in the sense that (1) holds if and only if

$$
s_{n}=\sum_{k=1}^{\infty} \alpha_{n k} \sigma_{k}
$$

then at least one divergent sequence $s_{n}$ is summable $A$ (such that $\sigma_{n}$ converges) if and only if the matrix $\alpha_{n k}$ fails to be conservative. The more general case in which the transformation (1) is reversible (in the sense of Mazur and Banach) was treated by J. D. Hill [1942]. The virtue of the criterion (5) lies in the fact that it is a simple criterion involving the matrix $a_{n k}$ itself; it may be used when $a_{n k}$ has no inverse, and when the inverse exists but is so complicated that the test for conservatism is not easily applied.

2. The condition (5). Each of the conditions (2), (3), and (4) has an obvious interpretation involving the matrix 


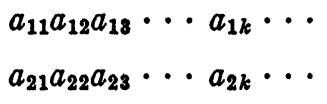

For example, (3) means that the elements of each column form a sequence converging to zero. The meaning of (5) is, roughly, that $\left|a_{n k}\right|$ is near zero whenever both $n$ and $k$ are large.

When a matrix satisfies (2), (3), and (4), there are several conditions which imply and are implied by (5). One is the condition (8) displayed below; it says, in other words, that the limit in (3) is uniform over the set $k=1,2,3, \cdots$.

3. A generalization of Theorem 1. While proving Theorem 1 , we can, without introducing complications, prove the following more general theorem.

THEOREM 3. If the matrix $a_{n k}$ of $A$ is such that

$$
\sum_{k=1}^{\infty}\left|a_{n k}\right|<\infty, \quad n=1,2,3, \cdots
$$

and

$$
\lim _{n \rightarrow \infty} \max _{x=1,2, \ldots}\left|a_{n k}\right|=0 \text {, }
$$

then there is at least one divergent sequence, whose elements are zeros and ones, which is summable $A$.

Actually, the proof will show clearly that there are "many" such sequences summable $A$.

4. Proof of Theorem 3. Let $a_{n k}$ be a matrix satisfying (7) and (8). We establish Theorem 3 by exhibiting a divergent sequence $s_{n}$, whose elements are all zeros and ones, which is summable $A$ to zero. To simplify typography, we sometimes write $a(n, k)$ and $s(k)$ for $a_{n k}$ and $s_{k}$. Let $\alpha(1), \alpha(2), \cdots$ be a sequence, of positive numbers, which converges to 0 so rapidly than $n \alpha(n) \rightarrow 0$; for example, let $\alpha(n)=n^{-2}$. Let $\beta(1), \beta(2), \cdots$ be a sequence, of positive numbers, which converges to 0 . The hypothesis (8) implies existence of an increasing sequence $n(1)<n(2)<\cdots$ of positive integers such that, for each $p=1,2, \cdots$,

$$
\left|a_{n, k}\right| \leqq \alpha(p),
$$$$
n \geqq n_{p}, k=1,2, \cdots \text {. }
$$

Such a sequence $n(p)$ being fixed, the hypothesis (7) implies that if 
$k(1), k(2), \ldots$ is a sequence of integers which becomes infinite sufficiently rapidly, then, for each $p=1,2,3, \cdots$,

$$
\sum_{k=k(p)+1}^{\infty}\left|a_{n, k}\right| \leqq \beta(p), \quad n_{p} \leqq n<n_{p+1} .
$$

Let a sequence $k(p)$ be fixed such that (10) holds and $k(p+1)>k(p)$ +1 for each $p=1,2, \cdots$. Let $s_{1}, s_{2}, \cdots$ be the particular sequence of zeros and ones defined by

$$
\begin{array}{rr}
s_{k}=1, & k=k_{1}, k_{2}, k_{3}, \cdots, \\
s_{k}=0 & \text { otherwise. }
\end{array}
$$

Then $s_{k}=1$ for an infinite set of $k$ 's and $s_{k}=0$ for an infinite set of $k$ 's; hence the sequence is divergent. Moreover the transform $\sigma_{1}, \sigma_{2}, \cdots$ of this sequence is such that, when $p=1,2,3, \cdots$ and $n_{p} \leqq n<n_{p+1}$,

$$
\begin{aligned}
\left|\sigma_{n}\right| & =\left|\sum_{k=1}^{\infty} a(n, k) s(k)\right|=\left|\sum_{j=1}^{\infty} a\left(n, k_{j}\right)\right| \\
& \leqq \sum_{j=1}^{p}\left|a\left(n, k_{j}\right)\right|+\sum_{j=p+1}^{\infty}\left|a\left(n, k_{j}\right)\right| \\
& \leqq \sum_{j=1}^{p} \alpha(p)+\sum_{k=k(p)+1}^{\infty}|a(n, k)|<p \alpha(p)+\beta(p) .
\end{aligned}
$$

Therefore, since $p \alpha(p) \rightarrow 0$ and $\beta(p) \rightarrow 0, \sigma_{n} \rightarrow 0$. Thus the particular divergent sequence of zeros and ones is summable $A$ to 0 and Theorem 3 is proved.

5. Relations between two methods of summability. Let $B$ and $C$ be two matrix methods of summability regular or not, of the form (1). It is the purpose of this section to discuss standard general procedures for determining relations between $B$ and $C$, and to show how Theorems 1 and 3 may be used. One says that $B$ includes $C$, or $B \supset C$, if $B\left\{s_{n}\right\}=C\left\{s_{n}\right\}$ whenever $C\left\{s_{n}\right\}$ exists; and that $B$ is stronger than $C$ if $B\left\{s_{n}\right\}$ exists whenever $C\left\{s_{n}\right\}$ exists, while $B\left\{s_{n}\right\}$ exists for at least one sequence for which $C\left\{s_{n}\right\}$ fails to exist. Two methods $B$ and $C$ are consistent if $B\left\{s_{n}\right\}=C\left\{s_{n}\right\}$ whenever both $B\left\{s_{n}\right\}$ and $C\left\{s_{n}\right\}$ exist, and are equivalent if $B\left\{s_{n}\right\}=C\left\{s_{n}\right\}$ whenever at least one of $B\left\{s_{n}\right\}$ and $C\left\{s_{n}\right\}$ exist.

For simplicity, we assume that $C$ is triangular and has an inverse; this means that $c_{n k}=0$ when $k>n$ and that $c_{n n} \neq 0$ for each $n=1,2, \ldots$. Let $a_{n k}$ be the matrix of the transformation $A$ defined by $A=B C^{-1}$. It is standard practice to use the fact that $B \supset C$ 
if and only if $A$ is regular, that is, if and only if (2), (3), and (4) hold. If, in a particular case, one shows that (2), (3), and (4) hold, one knows that $B \supset C$; the methods must then be consistent, but it remains unknown whether (i) $B$ and $C$ are equivalent or (ii) $B$ is stronger than $C$. If, in addition to (2), (3), and (4), one shows that (5) holds, then, by Theorem $1, B$ must be stronger than $C$.

There are cases in which, by reason of algebraic difficulties or by reason of actual failure of the conditions, one is unable to show that (2), (3), and (4) hold. (Those who work in the field know that the "norm condition" (2) is frequently the troublesome one.) It may nevertheless be possible to show that (7) and (8) hold. In such cases, Theorem 3 implies existence of sequences summable $B$ but nonsummable $C$.

\section{BiBLIOGRAPHY}

Agnew, R. P., 1932. On equivalence of methods of evaluation of sequences, Tohoku Math. J. vol. 35 (1932) pp. 244-252.

Hill, J. D., 1942. Some properties of summability, Duke Math. J. vol. 9 (1942) pp. 373-381.

Mercer, J., 1907. On the limits of real variants, Proc. London Math. Soc. (2) vol. 5 (1907) pp. 206-224.

Rad6, R., 1938. Some elementary Tauberian Theorems. I, Quart. J. Math. Oxford Ser. vol. 9 (1938) pp. 274-282.

Steinhaus, H., 1911. Quelques remarques sur la generalization de la notion de limite (in Polish), Prace Matematyczno-fizyczne vol. 22 (1911) pp. 121-134.

Sunouchi, G., 1934. On a linear transformation of infinite sequences, Proceedings of the Physico-Mathematical Society of Japan (3) vol. 16 (1934) pp. 161-163.

Toeplitz, O., 1911. Über allgemeine lineare Mittelbildungen, Prace Matematycznofizyczne vol. 22 (1911) pp. 113-119.

Cornell University 